\title{
Characteristics of Schumann Resonance Parameters at Kuju Station
}

\author{
Akihiro Ikeda ${ }^{1, *}$, Teiji Uozumi ${ }^{2}$, Akimasa Yoshikawa ${ }^{3}$, Akiko Fujimoto ${ }^{2}$, Shuji $\mathrm{Abe}^{2}$, \\ Hiromasa Nozawa $^{1}$, and Manabu Shinohara ${ }^{1}$ \\ ${ }^{1}$ National Institute of Technology Kagoshima College, Kirishima, Japan \\ ${ }^{2}$ International Center for Space Weather Science and Education, Kyushu University, Fukuoka, Japan \\ ${ }^{3}$ Department of Earth and Planetary Sciences, Kyushu University, Fukuoka, Japan
}

\begin{abstract}
The ground magnetic field variation in the extremely low frequency (ELF) range has been measured by an induction magnetometer at Kuju, Japan (KUJ; M.Lat. = 23.4 degrees, M. Lon. $=201.0$ degrees) since 2003. The first mode of the Schumann resonance (SR) around $8 \mathrm{~Hz}$ can be seen at KUJ. The SR in H (horizontal northward component) shows maximum peaks around 08 UT and 15 UT. In the case of D (horizontal eastward component), the SR shows its maximum peak around 08 UT. These peaks are coincident with the enhancement of lightning activity in Africa and Asia. Thus, we found the influence of the lightning activity on the observed SR at KUJ.
\end{abstract}

\section{Introduction}

The Schumann resonance (SR), whichwas detected by Blaser and Wagner [1960, 1962] for the first time, is the global resonance of electromagnetic waves generated by the global lightning activity.The resonance is formed by the Earth-ionosphere cavity and the specific resonance frequencies, which are about $8,14,21$, and $26 \mathrm{~Hz}$, appear in the ground magnetic field variation.

The diurnal variations of SR parameters reflect the properties of both the global lightning activity and the state of the Earth-ionosphere cavity. The variation in the amplitude of SR in a day show peaks which correspond with the three major thunderstorm centers, namely, African, Asian, and American [e.g., Sátori et al., 1996;Zhou et al., 2013].

In addition, SR parameters connect with the earth's climate [e.g., Williams, 1992; Price, 2000]. Williams [1992] suggested that the variation of SR frequency indicates the variations in the tropical temperature. Thus, the variation of SR can be used to monitor the earth's climate.

In order to use the SR parameters for monitoring theearth's climate, we need a better understanding of the SR detected at our observatory Kuju, Japan (KUJ; M.Lat. $=23.4$ degrees, M. Lon. $=201.0$ degrees). We present the effects of the global lightning activity to the fundamental mode of the SR at KUJ.

\footnotetext{
${ }^{\star}$ Corresponding author: a-ikeda@kagoshima-ct.ac.jp
} 


\section{Data Set}

The ground magnetic field variation in the extremely low frequency (ELF) range has been measured by an induction magnetometer at KUJ since 2003. The observation is a part of activities by International Center for Space Weather Science and Education, Kyushu University. The components of ground magnetic field used in this study are horizontal northward component $(\mathrm{H})$ and horizontal eastward component (D). The sampling rate of the ground magnetic field data is $50 \mathrm{~Hz}$. In this study, we examined the magnetic field data obtained from 2003 to 2012.

To identify SR parameters, which are frequency and power of the fundamental mode of the SR, we calculated PSD (power spectral density) every 10 seconds segment by using FFT (First Fourier Transformation). Since, the fundamental mode of SR is about $7.8 \mathrm{~Hz}$, we find a peak frequency ranging $6.0 \mathrm{~Hz}$ to $9.0 \mathrm{~Hz}$ for running average of the PSD data. Then
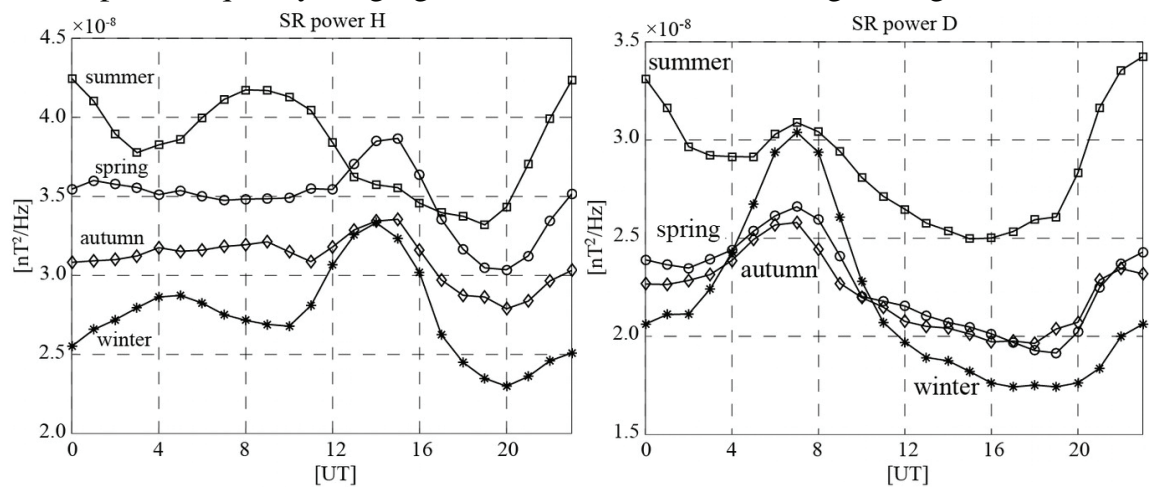

Fig. 2.The mean daily variation of SR power for spring (March, April, and May), summer (June, July, and August), autumn (September, October, and November), and winter (December, January, and February).
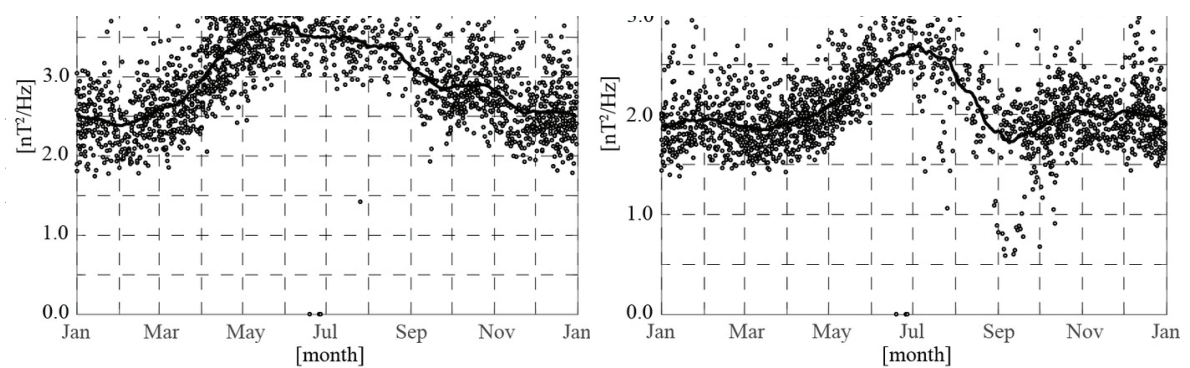

Fig. 1.The daily value of the SR power for $\mathrm{H}$ and $\mathrm{D}$ as a function of the day number. The lines indicate 30-day-centered moving average of the data.

is buried. The peak at 08 UT corresponds with the peak time of the lightning in Asia. Thus, the SR at Kuju indicates that the lightning activity from Africa affects through the year and the lightning activity from Asian center becomes stronger in summer.In the case of $\mathrm{D}$, the most prominent peak at 07 UT seems to be associated with the lightning activity from Asian center. While the peaks at 23 UT are noticeable both in H and D, they bear no reference to the three major regions of lightning activity.

As stated above, we found the response of the SR power at Kuju to the global lightning activity. The variation of the SR power seems to be affected by the thunderstorm regions which become active in relation to the observation site. However, the relationship between 
the peak at 23 UT and the global lightning activity is unclear.Setman and Fraser [1991] suggested that the local height of the ionospheric D region for a given observation site also affects the SR amplitude. In addition, Melnikov et al. [2004] indicated the terminator effect on the SR, and concluded that the effect is caused by the lateral ionospheric gradient across the terminator region. Thus, we need to take into account the state of the local ionosphere for the better understanding.

\section{Acknowledgement}

The ELF-range magnetic field data is taken by International Center for Space Weather Science and Education, Kyushu University. The authors wish to thank Dr. Rehman, A. U. and Mr. S. Abematsu for their supportive comments on an earlier draft of this paper.

\section{References}

1. Balser, M., Wagner C. A., Observation of earth-ionosphere cavity resonances, Nature, 188, 638-641, 1960.

2. Balser, M., Wagner, C. A., On frequency variations of the earth-ionosphere cavity modes, J. Geophys. Res. 67, 4081-4083, 1962.

3. Sátori, G., and B. Zieger, Spectral characteristics of Schumann resonances observed in Central Europe, J. Geophys. Res. 101, D23, 29,663-29,669, 1996.

4. Zhou, H., H. Yu, B. Cao, and X. Qiao, Diurnal and seasonal variations in the Schumann resonance parameters observed at Chinese observatories, Journal of Atmospheric and Solar-Terrestrial Physics, 98, 86-96, 2013.

5. Williams, E. R., The Schumann resonance: a global tropical thermometer, Science, 256 (5060), 1184-1187, 1992.

6. Price, C., Evidence for a link between global lightning activity and upper tropospheric water vapor, Nature, 406 (6793), 290-293, 2000.

7. Whipple, F. J. W., and E. J. Scrase, Point discharge in the electric field of the earth, Met. Off. Geophys. Mem., London, 68, 20, 1936.

8. Setman, D.D., and B. J. Fraser, Simultaneous observations of Schumann resonances in California and Australia: Evidence for intensity modulation by the local height of the D region, J. Geophys. Res. 96, 15,973-15,984, 1991.

9. Melnikov, A., C. Price, G. Sátori, and M. Füllekrug, Influence of solar terminator passages on Schumann resonance parameters, Journal of Atmospheric and SolarTerrestrial Physics, 66, 1187-1194, 2004. 